\title{
Statins as a New Therapeutic Perspective in Myocarditis and Postmyocarditis Dilated Cardiomyopathy
}

\author{
Editorial to "Pitavastatin Regulates Helper T-Cell Differentiation and Ameliorates \\ Autoimmune Myocarditis in Mice" by K. Tajiri et al.
}

\author{
Pietro Enea Lazzerini • Pier Leopoldo Capecchi • \\ Franco Laghi-Pasini
}

Published online: 9 July 2013

(C) Springer Science+Business Media New York 2013

Myocarditis is an inflammatory disease of the myocardium characterized by mononuclear cell infiltration with myocyte necrosis and degeneration [1].

The disease represents a relevant social problem worldwide, as it is one of the leading causes of dilated cardiomyopathy (DCM), particularly in young, previously healthy, individuals [2]. In fact, an underlying myocarditis is found in about $10 \%$ of patients with unexplained heart failure, and follow-up studies in subjects with acute myocarditis have reported the occurrence of DCM in $21 \%$ of the cases over a period of 3 years [1, 2]. Moreover, the disease is associated with a high arrhythmic risk, as demonstrated by post-mortem data identifying myocarditis in up to $12 \%$ of cases of sudden death in patients under 40 years of age [3].

Viruses are the most common primary cause of myocarditis in Western countries, predominantly enteroviral coxsackievirus B3 (CVB3), adenovirus, parvovirus B19, and human herpes virus 6 [1]. Although the pathogenesis of the disease remains largely unclear, there is substantial evidence that a virus-triggered autoimmune response to heart antigens, particularly cardiac myosin, critically contributes to the development and progression of myocarditis [1,2]. On this basis, two animal models of experimental myocarditis have been developed and currently used for studying the disease, i.e. infection-induced myocarditis, such as CVB3 infection, and experimental autoimmune myocarditis (EAM) induced by cardiac myosin immunization [2]. These models have significantly contributed to increase our knowledge of the pathogenesis of the disease, schematically consisting of three phases: (i) an acute phase, characterized by acute injury of the

P. E. Lazzerini $(\bowtie) \cdot$ P. L. Capecchi $\cdot$ F. Laghi-Pasini

Department of Medical Sciences, Surgery and Neurosciences, University of Siena, Siena, Italy

e-mail: lazzerini7@unisi.it cardiomyocytes induced by virus replication, (ii) a subacute phase, critically driven by a CD4+ T cell-mediated immune response triggered by the exposure of intracellular antigens as a consequence of myocyte injury, and (iii) a chronic phase, characterized by myocardial repair and remodelling, with the possible progression to DCM $[1,2,4]$. In this scenario, it is widely accepted that the mainstay actor is the T-helper (Th)1 cell, crucially involved in orchestrating the immune response required for the defence of the virus-infected heart, but also responsible for the organ damage [4]. Nevertheless, increasing evidence strongly suggests that also interleukin (IL)-17 producing Th17 cells play a key role in the process, particularly in the progression of myocarditis to DCM $[5,6]$.

On the basis of the increasing evidence for the involvement of the immune system in the pathogenesis of the disease, more than 20 treatment clinical trials using immunosuppressive, immunomodulating or anti-inflammatory agents as well as immunoadsorption therapy have been conducted [1]. However, although some of these studies demonstrated a benefit, particularly in chronic, virus-negative inflammatory cardiomyopathy, no standard management strategies targeting the immune-mediated pathogenesis of the disease could be defined as yet. As a consequence, current standard treatment schemes in myocarditis remain limited to the therapy of heart failure in most cases, and this fact is absolutely not satisfactory [1].

Statins are a widely employed class of cholesterol-lowering drugs acting by inhibiting the hydroxy-methyl-glutaryl Coenzyme A (HMG-CoA) reductase, a key enzyme involved in the conversion of HMG-CoA in the cholesterol precursor mevalonate [7, 8]. A large body of evidence demonstrated that statins reduce the cardiovascular risk to a greater extent to that expected on the basis of the blood cholesterol-lowering effect alone, as a result of a broad range of adjunctive activities, collectively known as pleiotropic effects, mainly mediated by 


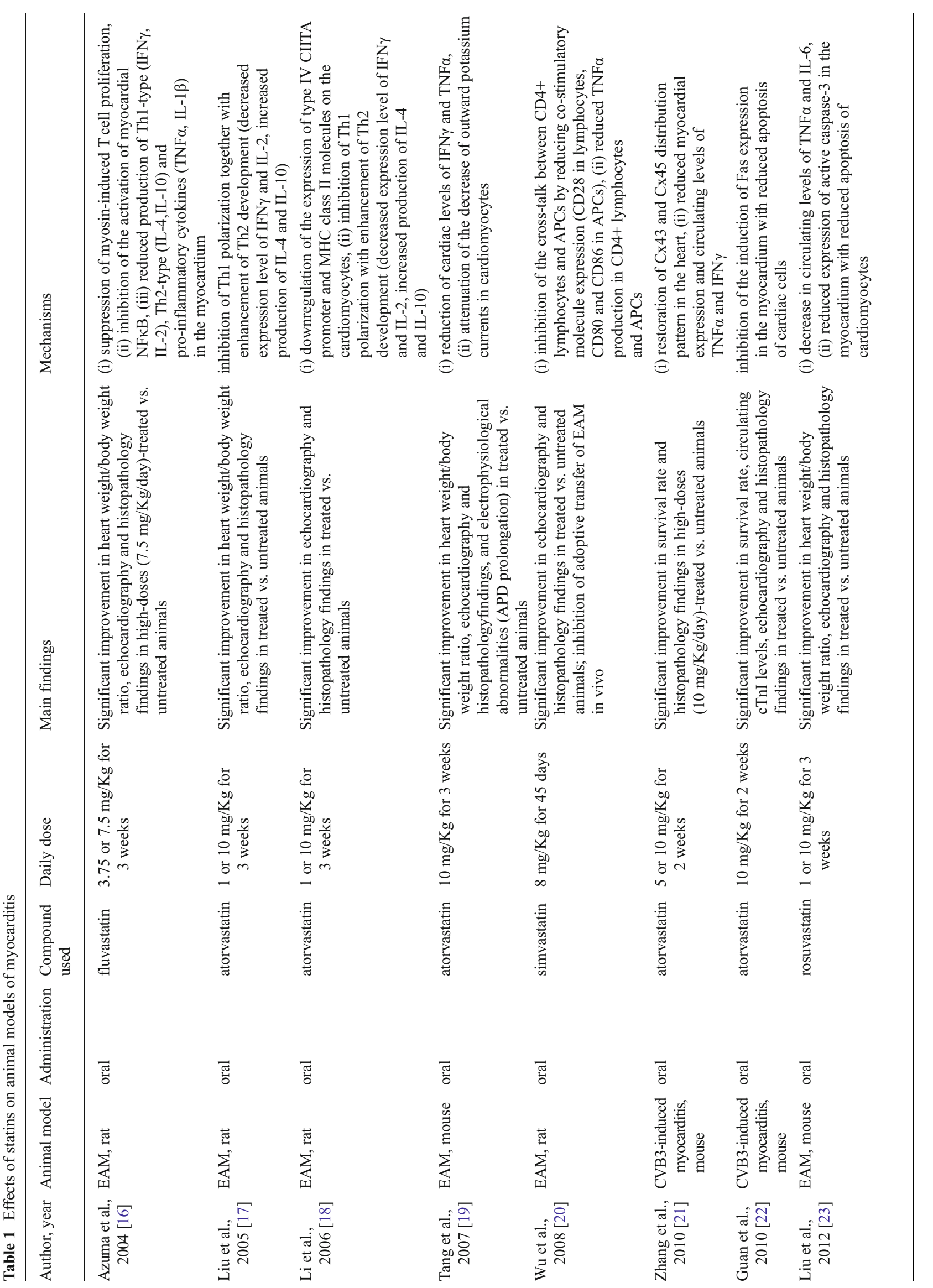


the interference on the protein (iso)prenylation processes [7]. In fact, mevalonate also represents the precursor of the isoprenoid derivatives, such as farnesyl pyrophosphate (FPP), and geranylgeranyl pyrophosphate (GGPP), which are involved in the regulation of several cellular mechanisms (signal transduction, cell proliferation and differentiation, cytoskeletal assembly and vescicle transport) by adding to specific intracellular proteins of the Ras, Rho, and Rab families [9]. Mounting evidence from basic and clinical studies has been demonstrating that the pleiotropic effects of statins include a wide variety of anti-inflammatory and immunomodulating properties that besides participating in the reduction of the cardiovascular risk, may also be useful in the treatment of some autoimmune diseases, particularly rheumatoid arthritis [10] and multiple sclerosis [11].

Notably, many recent in-vitro and in-vivo studies showed that statins are able to deeply affect, directly or indirectly, the function of the T-lymphocyte. Indirect effects include inhibition of T-cell homing, migration, and activation by targeting leukocytes and endothelial cells (reduced expression of chemokines, chemokine receptors, and adhesion molecules), as well as antigen presenting cells, APCs (inhibition of MHC class II antigen and costimulatory molecules expression; interference in the intracellular mechanisms of antigen processing and presentation). On the other hand, statins can also directly influence the T-lymphocyte by a) inhibiting cell activation: direct binding and block of lymphocyte function associated antigen-1, LFA-1, and reduced expression of the activation markers CD69 and CD25; b) inhibiting cell proliferation: disruption of T-cell receptor, TCR, signalling cascade at the critical steps regulated by small Ras-like GTPase; c) modulating Th1/Th2 differentiation: suppression of signal transducer and activator of transcription-4, STAT-4, with inhibition of Th1 polarization [12-14]. Finally, recent works demonstrated how statins are also effective in antagonizing Th17-mediated response, not only by reducing IL- 6 and IL-23 production by APCs, thereby inhibiting Th17 cell differentiation, but also by directly suppressing the IL-17 gene expression and protein secretion in CD4+ cells [15].

On the basis of the above data and in consideration of the key role played by $T$ helper cells in the pathogenesis of myocarditis, in the last years several authors [16-24] investigated the therapeutic potential as well as the putative underlying mechanisms of statin administration in animal models of the disease (Table 1). The results of these studies, in which different molecules were used (mainly atorvastatin, but also fluvastatin, simvastatin, rosuvastatin and pitavastatin), clearly and consistently demonstrated the ability of this class of drugs to significantly improve both cardiac function, as assessed by echocardiography, and the histopathological severity of the disease, with respect to untreated animals. Moreover, these studies provided relevant information about some potential immunomodulatory mechanisms responsible for the beneficial 
effects of statins in myocarditis. In particular, the effectiveness of statins seems to be primarily related to a series of activities on the T-lymphocyte, including suppression of cell proliferation, inhibition of Th1 polarization, and interference in the cross-talk with APCs. In turn, these effects finally result in a marked reduction of the amount of cardiac Th1-type and proinflammatory cytokines, thus attenuating their harmful consequences on the myocardium, i.e. cardiomyocyte apoptosis, and structural and electrophysiological remodelling, which are chiefly involved in the severe depression of cardiac function, and the high arrhythmic risk characterizing myocarditis and inflammatory DCM [16-24].

In this issue of Cardiovascular Drugs and Therapy, Tajiri and colleagues [24] demonstrate that in BALB/c mice developing EAM after immunization with murine $\alpha$-myosin heavy chain, pitavastatin administration significantly reduces the pathophysiological severity of the disease by targeting the function of the T-cell. The paper, besides providing convincing data further supporting the view that statins are protective in this pathological condition, also gives new original information contributing to clarify the mechanisms of action by which these drugs counteract myocarditis development and may reduce the associated risk of progression to DCM.

In particular, the authors show for the first time that the beneficial effects of statins on EAM are associated not only with the previously reported inhibition of Th1 polarization, but also include a concomitant suppressive activity on Th17 differentiation, which seems to be even stronger as it occurs for doses lower than those required to inhibit the differentiation of Th1 cells. This effect may be of particular relevance in the clinical setting as increasing evidence indicates that although Th17 cells, and in particular IL-17A, have only a mild effect on the severity of myocarditis during the acute phase in which the major role seems to be played by the Th1 cell [2, 4], however they are essential for the long-term progression to DCM [5, 6]. In fact, it has been demonstrated that IL-17A-deficient mice, while developing myocarditis with similar incidence and severity to wild-type controls, were protected from postmyocarditis remodelling and did not develop DCM. Notably, these mice showed reduced interstitial myocardial fibrosis and downregulated expression and activity of matrixmetalloproteinases in the heart [5]. Moreover, treatment of $\mathrm{BALB} / \mathrm{c}$ mice with anti-IL17A monoclonal antibody administered after the onset of EAM abrogated myocarditis-induced cardiac fibrosis and preserved ventricular function [5]. These observations, together with the evidence that IL-17A is able to directly signal the function of the cardiac fibroblast [25-27], indicate that this cytokine critically contributes to myocardial fibrosis and remodelling of the extracellular matrix, thus driving the progression to DCM.

On this basis, the novel data of Tajiri and colleagues [24] suggest that by modulating the function of the T-cell, statins exert multistep beneficial effects in myocarditis, including suppression of the Th1-driven autoimmune response during the subacute phase as well as inhibition of the Th17-dependent postmyocarditis cardiac remodelling occurring in the chronic phase, thus potentially representing an attractive new therapy for the human disease.

At the moment, no clinical studies investigating the effects of statins in patients with myocarditis are available. Moreover, although more than 10 studies have been so far conducted in patients with established heart failure, including DCM, overall reporting the advantages of statin therapy [28], however they were in most cases small clinical studies and, particularly, only one of these involved patients with biopsy-proven inflammatory DCM [29]. In this randomized study including 74 patients, a 6 month-therapy with atorvastatin $(40 \mathrm{mg} /$ day $)$ was associated with a significant improvement in both clinical and echocardiographic parameters as compared to conventional treatment. Interestingly, a significant reduction in the frequency of T-cells and macrophages in myocardial infiltrates, paralleled with a decrease in the expression of the class II MHC antigens, was detected in repeated biopsies in the atorvastatin group, but not in controls [29].

In conclusion, mounting data from in-vivo studies provide evidence that statins are highly effective in reducing the severity of experimental myocarditis in animal models through a multi-level, sequential interference on the T-cell-mediated immune response critically involved in the development and the progression of the disease. Large randomized clinical trials are warranted and needed to verify whether this basic data may be transposed into the clinical setting, thus defining if statins may take a place in the currently unsatisfactory therapeutic strategy of patients with myocarditis and inflammatory DCM.

Conflict of Interest The authors declare that they have no conflict of interest.

\section{References}

1. Kindermann I, Barth C, Mahfoud F, et al. Update on myocarditis. J Am Coll Cardiol. 2012;59:779-92.

2. Corsten MF, Schroen B, Heymans S. Inflammation in viral myocarditis: friend or foe? Trends Mol Med. 2012;18:426-37.

3. Fabre A, Sheppard MN. Sudden adult death syndrome and other nonischaemic causes of sudden cardiac death. Heart. 2006;92:316-20.

4. Barin JG, Ciháková D. Control of inflammatory heart disease by CD4(+) T cells. Ann N Y Acad Sci. 2013;1285:80-96.

5. Baldeviano GC, Barin JG, Talor MV, et al. Interleukin-17A is dispensable for myocarditis but essential for the progression to dilated cardiomyopathy. Circ Res. 2010;106:1646-55.

6. Nindl V, Maier R, Ratering D, et al. Cooperation of Th1 and Th17 cells determines transition from autoimmune myocarditis to dilated cardiomyopathy. Eur J Immunol. 2012;42:2311-21.

7. Wierzbicki AS, Poston R, Ferro A. The lipid and non-lipid effects of statins. Pharmacol Ther. 2003;99:95-112.

8. Bhatnagar D, Soran H, Durrington PN. Hypercholesterolaemia and its management. BMJ. 2008;337:a993. 
9. Corsini A, Bellosta S, Baetta R, Fumagalli R, Paoletti R, Bernini F. New insights into the pharmacodynamic and pharmacokinetic properties of statins. Pharmacol Ther. 1999;84:413-28.

10. Lazzerini PE, Capechi PL, Selvi E, et al. Statins and the joint: multiple targets for a global protection? Semin Arthritis Rheum. 2011;40:430-46.

11. Willey JZ, Elkind MS. 3-Hydroxy-3-methylglutaryl-coenzyme A reductase inhibitors in the treatment of central nervous system diseases. Arch Neurol. 2010;67:1062-7.

12. Ghittoni R, Patrussi L, Pirozzi K, et al. Simvastatin inhibits T-cell activation by selectively impairing the function of Ras superfamily GTPases. FASEB J. 2005;19:605-7.

13. Ghittoni R, Napolitani G, Benati D, et al. Simvastatin inhibits the MHC class II pathway of antigen presentation by impairing Ras superfamily GTPases. Eur J Immunol. 2006;36:2885-93.

14. Ghittoni R, Lazzerini PE, Laghi-Pasini F, Baldari CT. T lymphocytes as targets of statins: molecular mechanisms and therapeutic perspectives. Inflamm Allergy Drug Targets. 2007;6:316 .

15. Zhang X, Markovic-Plese S. Statins' immunomodulatory potential against Th17 cell-mediated autoimmune response. Immunol Res. 2008;41:165-74.

16. Azuma RW, Suzuki J, Ogawa M, et al. HMG-CoA reductase inhibitor attenuates experimental autoimmune myocarditis through inhibition of T cell activation. Cardiovasc Res. 2004;64:412-20.

17. Liu W, Li WM, Gao C, Sun NL. Effects of atorvastatin on the Th1/ Th2 polarization of ongoing experimental autoimmune myocarditis in Lewis rats. J Autoimmun. 2005;25:258-63.

18. Li WM, Liu W, Gao C, Zhou BG. Immunoregulatory effects of atorvastatin on experimental autoimmune myocarditis in Lewis rats. Immunol Cell Biol. 2006;84:274-80.

19. Tang Q, Huang J, Qian H, et al. Antiarrhythmic effect of atorvastatin on autoimmune myocarditis is mediated by improving myocardial repolarization. Life Sci. 2007;80:601-8.
20. Wu JL, Matsui S, Zong ZP, et al. Amelioration of myocarditis by statin through inhibiting cross-talk between antigen presenting cells and lymphocytes in rats. J Mol Cell Cardiol. 2008;44:1023-31.

21. Zhang $\mathrm{A}$, Zhang $\mathrm{H}$, Wu S. Immunomodulation by atorvastatin upregulates expression of gap junction proteins in coxsackievirus B3 (CVB3)-induced myocarditis. Inflamm Res. 2010;59:255-62.

22. Guan J, Sun X, Liang Y, et al. Atorvastatin attenuates Coxsackie virus $\mathrm{B} 3 \mathrm{~m}$-induced viral myocarditis in mice. $\mathrm{J}$ Cardiovasc Pharmacol. 2010;56:540-7.

23. Liu X, Li B, Wang W, et al. Effects of HMG-CoA reductase inhibitor on experimental autoimmune myocarditis. Cardiovasc Drugs Ther. 2012;26:121-30.

24. Tajiri K, Shimojo N, Sakai S, et al. Pitavastatin regulates helper Tcell differentiation and ameliorates autoimmune myocarditis in mice. Cardiovasc Drugs Ther. 2013;27:this issue

25. Cortez DM, Feldman MD, Mummidi S, et al. IL-17 stimulates MMP1 expression in primary human cardiac fibroblasts via $\mathrm{p} 38$ MAPKand ERK1/2-dependent C/EBP-beta, NF-kappaB, and AP-1 activation. Am J Physiol Heart Circ Physiol. 2007;293:H3356-65.

26. Valente AJ, Yoshida T, Gardner JD, Somanna N, Delafontaine P, Chandrasekar B. Interleukin-17A stimulates cardiac fibroblast proliferation and migration via negative regulation of the dualspecificity phosphatase MKP-1/DUSP-1. Cell Signal. 2012;24:560-8

27. Liu Y, Zhu H, Su Z, et al. IL-17 contributes to cardiac fibrosis following experimental autoimmune myocarditis by a PKC $\beta /$ Erk $1 / 2 / \mathrm{NF}-\mathrm{kB}$ dependent signaling pathway. Int Immunol. 2012;24:605-12.

28. Bielecka-Dabrowa A, Mikhailidis DP, Hannam S, Aronow WS, Rysz J, Banach M. Statins and dilated cardiomyopathy: do we have enough data? Expert Opin Investig Drugs. 2011;20:315-23.

29. Wojnicz R, Wilczek K, Nowalany-Kozielska E, et al. Usefulness of atorvastatin in patients with heart failure due to inflammatory dilated cardiomyopathy and elevated cholesterol levels. Am J Cardiol. 2006;97:899-904. 